\title{
Similarity-Based Unsupervised Spelling Correction Using BioWordVec: Development and Usability Study of Bacterial Culture and Antimicrobial Susceptibility Reports
}

Taehyeong Kim ${ }^{1 *}$, BS; Sung Won Han ${ }^{1 *}$, PhD; Minji Kang ${ }^{1}$, BS; Se Ha Lee², BS; Jong-Ho Kim³ ${ }^{3}$ PhD; Hyung Joon $\mathrm{Joo}^{3}, \mathrm{MD}, \mathrm{PhD}$; Jang Wook Sohn ${ }^{4}, \mathrm{MD}, \mathrm{PhD}$

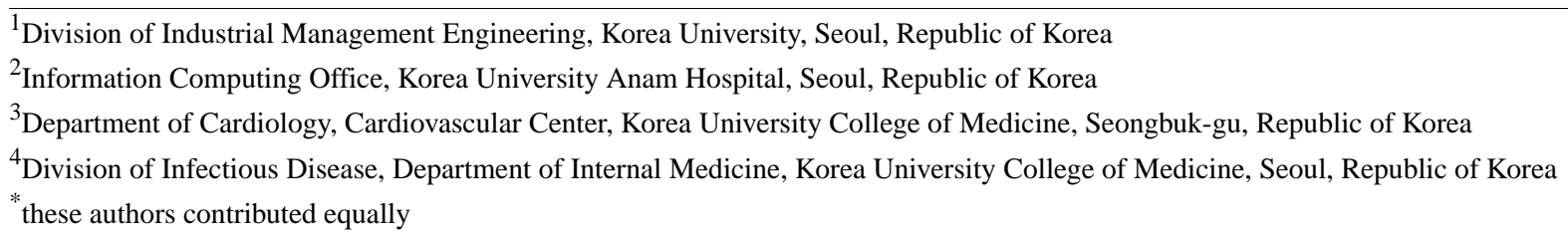

\section{Corresponding Author:}

Hyung Joon Joo, MD, PhD

Department of Cardiology

Cardiovascular Center

Korea University College of Medicine

73, Inchon-ro

Seongbuk-gu, 02841

Republic of Korea

Phone: 8229206411

Email:drjoohj@gmail.com

\section{Abstract}

Background: Existing bacterial culture test results for infectious diseases are written in unrefined text, resulting in many problems, including typographical errors and stop words. Effective spelling correction processes are needed to ensure the accuracy and reliability of data for the study of infectious diseases, including medical terminology extraction. If a dictionary is established, spelling algorithms using edit distance are efficient. However, in the absence of a dictionary, traditional spelling correction algorithms that utilize only edit distances have limitations.

Objective: In this research, we proposed a similarity-based spelling correction algorithm using pretrained word embedding with the BioWordVec technique. This method uses a character-level N-grams-based distributed representation through unsupervised learning rather than the existing rule-based method. In other words, we propose a framework that detects and corrects typographical errors when a dictionary is not in place.

Methods: For detected typographical errors not mapped to Systematized Nomenclature of Medicine (SNOMED) clinical terms, a correction candidate group with high similarity considering the edit distance was generated using pretrained word embedding from the clinical database. From the embedding matrix in which the vocabulary is arranged in descending order according to frequency, a grid search was used to search for candidate groups of similar words. Thereafter, the correction candidate words were ranked in consideration of the frequency of the words, and the typographical errors were finally corrected according to the ranking.

Results: Bacterial identification words were extracted from 27,544 bacterial culture and antimicrobial susceptibility reports, and 16 types of spelling errors and 914 misspelled words were found. The similarity-based spelling correction algorithm using BioWordVec proposed in this research corrected 12 types of typographical errors and showed very high performance in correcting $97.48 \%$ (based on F1 score) of all spelling errors.

Conclusions: This tool corrected spelling errors effectively in the absence of a dictionary based on bacterial identification words in bacterial culture and antimicrobial susceptibility reports. This method will help build a high-quality refined database of vast text data for electronic health records. 


\section{KEYWORDS}

spelling correction; natural language processing; bacteria; electronic health record

\section{Introduction}

\section{Background}

Among various industries, the medical industry produces many unstructured forms of examination reports. It is very important to establish a structured form of accurate medical documentation to provide accurate diagnoses and treatments to patients [1]. False medical information because of spelling errors can lead to medical and/or treatment errors, resulting in serious risks for patients. For example, errors in the spelling of organism names or drugs with similar spelling in bacterial culture tests have negative effects on not only the diagnosis and treatment of patients, but also the management of infectious diseases and nosocomial infections in hospitals.

While many patient electronic health records are documented in a structured form, the bacterial culture report is still stored as images or as an unrefined text data form in most hospitals. Mapping terms for bacterial identification are necessary to proceed with medical data studies, such as detection and diffusion path studies of infectious diseases. However, since large-scale clinical text data are mostly written by doctors or semiautomatic systems, there can be problems with data consistency, typographical errors, and stop words [2].

In clinical text data, the extraction-transformation-load (ETL) process for medical terms is typically performed through exact string matching of words that appear in the dictionary. However, words not present in the dictionary or severely misspelled words have difficulty matching to terms. Because medical terms are complex and field specific, this problem makes it difficult to apply the same general data refining methods [3]. Rule-based spelling correction algorithms cannot ensure the accuracy and reliability of the data because of incorrect data preprocessing. This method also has to check all test results and find the errors directly, resulting in a considerable cost problem.

\section{Related Work}

\section{Spelling Correction in the Medical Domain}

It is very difficult to construct dictionaries for all medical terms and abbreviations. A related study of spelling correction algorithms specialized in medical record text data was conducted. Lai et al [4] proposed a noisy channel-based spelling check algorithm for medical text. Named entity recognition (NER) was used to achieve an error detection performance of up to $94.4 \%$ with a spelling correction accuracy of up to $88.2 \%$, producing high performance spelling correction results in various clinical documents. Fivez et al $[5,6]$ proposed a spelling check algorithm for clinical free text using fastText of the $\mathrm{N}$-gram embedding technique. After generating misspelled words in MIMIC-III [7] to measure similarity with the candidate group that fits the context, the similarity was ranked using the Damerau-Levenshtein distance. This method suggested a way to solve the out-of-vocabulary (OOV) problem in clinical data.

\section{Subword-Level Word Vector Representation}

Traditional spelling correction algorithms using edit distance or pronunciation algorithms have limitations in correcting word-level issues that fit the context. There are subword-level embedding methods for learning concurrent word information to consider context understanding. FastText [8] expresses a word by the sum of the N-gram vector of the character level. The embedding method at the subword level solves the disadvantages that involve difficulty in application to languages with varying morphological changes or low frequency. This method was strong at solving the OOV problem, and accuracy was high for rare words in the word set. BioWordVec [9] learns clinical record data from PubMed and MIMIC-III clinical databases using fastText. Based on 28,714,373 PubMed documents and 2,083,180 MIMIC-III clinical database documents, the entire corpus was built. The Medical Subject Headings (MeSH) term graph was organized to create a heading sequence and to carry out word embedding based on a sequence combining $\mathrm{MeSH}$ and PubMed. BioWordVec provided a 200-dimensional pretrained word embedding matrix.

\section{Limitations With Existing Approaches}

The method proposed by Lai et al [4] has a limitation in that spelling corrections are not made in the absence of a dictionary. The method proposed by Fivez et al [5,6] solves the OOV problem, but has a similar limitation in that spelling corrections are not made in the absence of a dictionary.

\section{Our Approach}

This paper proposes a similarity-based spelling correction algorithm through pretrained word embedding in medical field data. Using the BioWordVec model of the character level, which has pretrained clinical record data from the MIMIC-III clinical database, the model progresses learning on spelling corrections end-to-end. The proposed model has the advantage of being able to make spelling corrections in the absence of a dictionary. In addition, it is effective against new types of typographical errors that may occur in the future, and it is highly utilized in the field because it uses unsupervised learning that does not require direct label assignment. We aimed to use this model to develop a spelling correction system suitable for various types of medical text data.

\section{Methods}

\section{Data Set}

\section{Bacterial Culture and Antimicrobial Susceptibility Reports}

In this study, the bacterial culture and antimicrobial susceptibility reports from Korea University Anam Hospital, Korea University Guro Hospital, and Korea University Ansan Hospital were used. The bacterial culture and antimicrobial susceptibility report data were collected for 17 years (from 2002 to 2018), and in each year, reports for 1 month were used for the experiment. In total, 180,000 items were retrieved, with 
27,544 having meaningful test results. Using the self-developed rule-based ETL algorithm [10], unstructured bacterial culture and antimicrobial susceptibility reports were converted into structured text data. After preprocessing through lexical processing, such as sentence segmentation, tokenization, and stemming using regular expressions, there were 320 types of bacterial identification words in the report. Among the extracted bacterial identification words, 16 types of spelling errors and 914 misspelled words were found. Table 1 presents the typographical errors based on their occurrence.

Table 1. Misspelling frequency table.

\begin{tabular}{ll}
\hline Misspelling & Occurrence, $\mathrm{n}$ \\
\hline staphylococcus & 827 \\
sstreptococcus & 21 \\
adecarboxylate & 19 \\
parpinfluenzae & 18 \\
papatyphi & 7 \\
pseudodiphthericum & 6 \\
urealyticm & 5 \\
chromogens & 2 \\
flavbacterium & 2 \\
ferentum & 1 \\
koneensis & 1 \\
ochrobacterium & 1 \\
orytihabitans & 1 \\
shingobacterium & 1 \\
stacherbrandfii & 1 \\
perosis & 1 \\
\hline
\end{tabular}

\section{Methodology}

\section{Misspelling Detection}

Systematized Nomenclature of Medicine (SNOMED) clinical terms (CT) [11] is a set of systematically structured medical terms used in medical clinical documents and reports. It is the world's largest multilingual clinical terminology system. In the corpus constructed by tokenizing the bacterial identification result reports, words that were not mapped to SNOMED CT were defined and detected as typographical errors [12].

\section{Candidate Generation}

Using the fastText [8] technique, prelearned word embedding was used to generate a group of corrected word candidates with high similarity considering the edit distance. In this study, the BioWordVec [9] model that was prelearned from the clinical database was used.

The number of words that were most similar, cosine similarity, and edit distance were set as hyperparameters for generating a correction candidate group. In addition, constraints for candidate words were used based on the dictionary constructed for the existing general terms, the length of the word, and the frequency of the word. In this study, the number of most similar words was set to 30 , cosine was set to 0.80 , and edit distance was set to 3 as hyperparameters.

Character-based spell checking algorithms were used to determine edit distances to generate or rank candidate groups.
The Levenshtein edit distance [13] is the number of operations required to convert one word into another. It can find the minimum editing distance that considers the insertion, deletion, replacement, and transposition (replacement of two adjacent characters) for most spelling errors. The model proposed in this paper uses the Damerau-Levenshtein distance [14] as the edit distance. The formula is as follows:

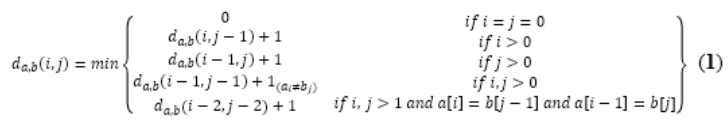

\section{Candidate Ranking}

The final correction word is suggested by ranking the correction candidate groups. The pretrained word embedding was learned by the fastText technique, and the vocabulary was sorted in descending order according to frequency. The methodology proposed in this study has two assumptions. First, in clinical databases, correctly spelled words may appear relatively more frequently than misspelled words [15]. Second, the larger the corpus used for learning, the greater the frequency of correctly spelled words [15]. The BioWordVec [9] model used in this research can sufficiently satisfy the above two assumptions.

The model proposed in this research limited the search for the range of the most similar words. Through a grid search, a similarity-based candidate group that considers the frequency of words was proposed [16]. After sorting the ranking of the 
generated correction candidate words based on similarity, typographical errors can be corrected.

\section{Overall Architecture}

Figure 1 shows the architecture of the spelling correction algorithm proposed in this paper.

Figure 1. Similarity-based unsupervised spelling correction architecture. SNOMED: Systematized Nomenclature of Medicine.

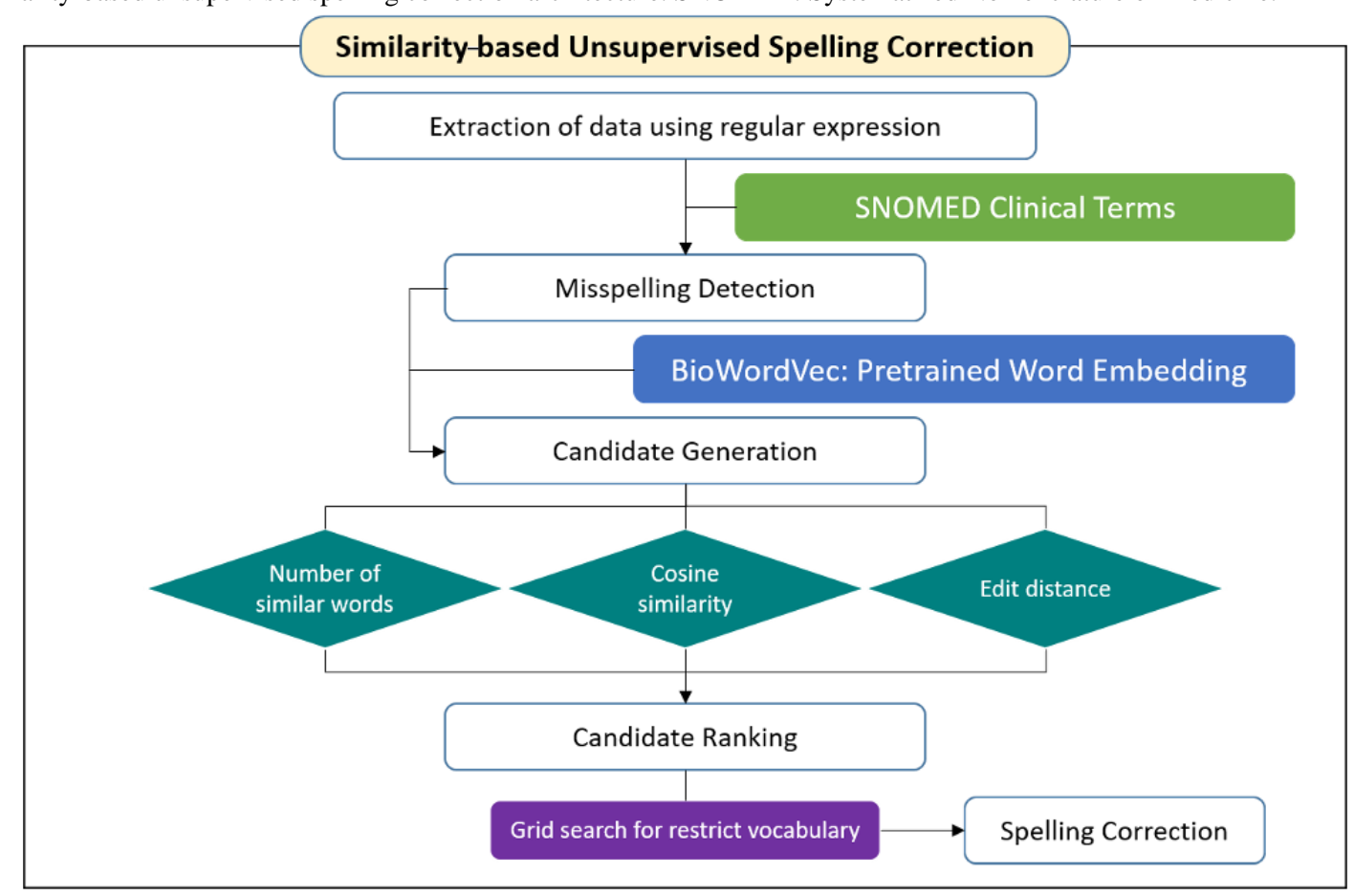

\section{Results}

\section{Experiments}

A typographical error that appears in bacterial culture and antimicrobial susceptibility reports is a word that can be corrected within three edit distances, as shown in Table 2. Most typographical errors have a correctly spelled word within one edit distance. Therefore, in the model proposed in this study, the critical value of the editing distance for generating the correction candidate group was set to 3 or less.

Table 2. Correction table using edit distance.

\begin{tabular}{|c|c|}
\hline Correction & Edit distance \\
\hline stapylococcus to staphylococcus & 1 \\
\hline sstreptococcus to streptococcus & 1 \\
\hline adecarboxylate to adecarboxylata & 1 \\
\hline parpinfluenzae to parainfluenzae & 1 \\
\hline pseudodiphtericum to pseudodiphtheriticum & 2 \\
\hline urealyticm to urealyticum & 1 \\
\hline chromogens to chromogenes & 1 \\
\hline flavbacterium to flavobacterium & 1 \\
\hline koneensis to koreensis & 1 \\
\hline ochrobacterium to ochrobactrum & 2 \\
\hline orytihabitans to oryzihabitans & 1 \\
\hline shingobacterium to sphingobacterium & 1 \\
\hline stacherbrandfii to stackebrandtii & 3 \\
\hline perosis to peroris & 1 \\
\hline
\end{tabular}




\section{Comparison of Pretrained Embeddings}

All of the pretrained word embeddings used in this study were learned based on the fastText methodology, and the corpus was constructed without distinction between spelling errors and correct spelling during learning. To compare the performance of the BioWordVec model introduced in the previous study, four pretrained embeddings provided by Facebook were used.

The following are the five pretrained embeddings: (1) BioWordVec, 200-dimensional embedding vectors learned using fastText for PubMed and MIMIC-III; (2) English word vectors, 300-dimensional embedding vectors learned using fastText for general text and from Wikipedia; (3) Crawled English subword vectors, 300-dimensional embedding vectors learned using fastText for the 2,000,000 lower words that appear in English word documents; (4) Wiki word vectors, 300-dimensional embedding vectors learned using fastText in
Wikipedia; (5) Simple Wiki word vectors, 300-dimensional embedding vectors learned using fastText in Simple Wikipedia.

The cosine similarity of all models was set to 0.80 or higher, the editing distance threshold was set to 3 or less, and the most similar words were tested under the same conditions with 30 words. The evaluation index is the exact spelling of the total 16 typographical errors that appear in the bacterial assimilation report with correction rate. Table 3 shows the rate of correction for typographical errors according to pretrained embeddings.

The spelling correction algorithm using BioWordVec showed very high performance compared to the performance of the other pretrained word embedding models. The methodology proposed in this study has the advantage of being used even in the absence of a dictionary. However, it was confirmed that pretrained word embedding based on the clinical database is necessary to correct errors in the bacterial identification report.

Table 3. Comparison of pretrained embedding.

\begin{tabular}{ll}
\hline Pretrained embedding model & Correction rate \\
\hline BioWordVec & 0.75 \\
English word vectors & 0.00 \\
Crawled English subword vectors & 0.00 \\
Wiki word vectors & 0.31 \\
Simple Wiki word vectors & 0.19 \\
\hline
\end{tabular}

\section{Evaluation}

Through a comparative experiment as shown in Table 3, it is possible to correct typographical errors using pretrained word embedding without building a dictionary. To evaluate the performance of the model proposed in this study, its performance was compared with a rule-based spelling correction algorithm [17] using a dictionary and a situation without spelling correction. SymSpell [18] was used as a spelling correction algorithm based on the edit distance rule.

SymSpell [18] can correct typographical errors 1 million times faster than rule-based spelling correction [17] and can use existing dictionaries through a symmetric deletion spelling correction algorithm. SymSpell uses the Damerau-Levenshtein edit distance [14], which was set to 3 for the experiment under the same conditions as the model proposed in this study. SCOWL [19] and Dorland medical dictionary [20,21] were used as dictionaries for SymSpell, and a total of 100,000 correct word dictionaries were constructed.

Table 4 shows the evaluation results through the NER task that extracts the bacterial identification words. In the table, accuracy is the number of words corrected for all misspellings. Precision is the proportion of corrected words that the actual corrections match exactly. Recall is the proportion of correct corrected words among actual typographical errors. F1 score is the harmonic mean of precision and recall. SUSC (similarity-based unsupervised spelling correction) in Table 4 is the model proposed in this study.

In this study, the similarity-based spell checking algorithm SUSC using BioWordVec corrected 12 types of typographical errors and showed very high performance in correcting $97.48 \%$ (based on F1 score) of all spelling errors. Both models were able to correct frequent typographical errors, so the overall correction rate was high. However, since SymSpell only corrects certain words, the F1 score showed little difference compared with the nonspelling situation. The Dorland medical dictionary was not able to fully understand bacterial identification names for infectious diseases, and the rule-based spell checking algorithms using edit distance did not work well according to the established dictionaries. Constructing an accurate dictionary that can be used in a rule-based spell checking algorithm is very expensive and time consuming.

Table 4. Model performance using BioWordVec.

\begin{tabular}{llll}
\hline Model & Accuracy & Precision & Recall \\
\hline No spelling correction & 0.98 & 0.94 & 0.93 \\
SymSpell & 1.00 & 0.94 & 0.94 \\
SUSC $^{\text {a }}$ (BioWordVec) & 1.00 & 0.97 & 0.94 \\
\hline
\end{tabular}

${ }^{\text {a }}$ SUSC: similarity-based unsupervised spelling correction. 


\section{Comparison of Similarity}

Using the SUSC model proposed in this study, the degree of similarity of words depending on correction was examined. Table 5 shows the similarity of words according to whether they are corrected.

As shown in Table 5, typographical errors that were not corrected with the correct spelling have low cosine similarity with the correctly spelled word as a whole. In the case of nonword errors, which involve words that do not actually exist, most of the words were corrected accurately. Miscorrected typographical errors included real-word errors where the word actually exists but is not appropriate for grammar or context. Since real-word errors are determined to be similar in meaning to words that do not fit the situation, the cosine similarity is relatively low for the word vector to be corrected. The model proposed in this study has the advantage of quantitatively expressing the relative distance between typographical errors and correctly spelled words by utilizing the similarity between words. Through the proposed model, it is possible to compare and determine whether the error detected with the framework is actually a typographical error that can occur often.

Table 5. Comparison of similarity according to correction.

\begin{tabular}{|c|c|c|}
\hline Change & Correction & Similarity \\
\hline adecarboxylate to adecarboxylata & Corrected & 0.90 \\
\hline flavbacterium to flavobacterium & Corrected & 0.83 \\
\hline koneensis to koreensis & Corrected & 0.87 \\
\hline ochrobacterium to ochrobactrum & Corrected & 0.93 \\
\hline orytihabitans to oryzihabitans & Corrected & 0.90 \\
\hline papatyphi to paratyphi & Corrected & 0.89 \\
\hline parpinfluenzae to parainfluenzae & Corrected & 0.86 \\
\hline pseudodiphtericum to pseudodiphtheriticum & Corrected & 0.93 \\
\hline shingobacterium to sphingobacterium & Corrected & 0.93 \\
\hline sstreptococcus to streptococcus & Corrected & 0.95 \\
\hline stapylococcus to staphylococcus & Corrected & 0.88 \\
\hline urealyticm to urealyticum & Corrected & 0.84 \\
\hline chromogens to chromogenes & Not corrected & 0.71 \\
\hline ferentum to fermentum & Not corrected & 0.47 \\
\hline perosis to peroris & Not corrected & 0.42 \\
\hline stacherbrandfii to stackebrandtii & Not corrected & 0.59 \\
\hline
\end{tabular}

\section{Discussion}

It is difficult to compare our results with previous results because the system implementation and data set used in the related work are not publicly available. The model proposed in this research was capable of spelling correction through unsupervised learning, but it lacked the performance required for infrequent typographical errors and real-word errors. In addition, there was a problem of randomly setting the reference values for cosine similarity and edit distance when creating a correction candidate group. Methods should be devised to establish appropriate thresholds for hyperparameters through experiments.

This research proposes a similarity-based spelling correction algorithm using pretrained word embedding to extract correct medical terminology from unstructured text data related to infectious diseases. The suggested algorithm has the advantage of being able to check spelling and make corrections in the absence of a correct spelling dictionary. In addition, it solves the OOV problem and can modify words based on context.

As a result of the experiments conducted in this research, we were able to detect and correct spelling errors in the absence of a dictionary for bacterial terms appearing in bacterial culture and antimicrobial susceptibility reports. Our model efficiently refined and processed large medical text data. It has been proven experimentally that it is a method suitable for processing natural language involving high expertise and complexity, such as medical terminology. Ideally, the results of this research will serve as a foundation to build vast amounts of text data in electronic health records into high-quality databases.

\section{Acknowledgments}

This research was supported by a grant from the Korea Health Technology R\&D Project through the Korea Health Industry Development Institute (KHIDI), funded by the Ministry of Health \& Welfare, Republic of Korea (grant number: HI19C0201, HI19C0360). This research was also supported by Brain Korea 21 FOUR. 


\section{Conflicts of Interest}

None declared.

\section{References}

1. Wong W, Glance D. Statistical semantic and clinician confidence analysis for correcting abbreviations and spelling errors in clinical progress notes. Artif Intell Med 2011 Nov;53(3):171-180. [doi: 10.1016/j.artmed.2011.08.003] [Medline: 21924593]

2. Hersh WR, Campbell EM, Malveau SE. Assessing the feasibility of large-scale natural language processing in a corpus of ordinary medical records: a lexical analysis. Proc AMIA Annu Fall Symp 1997:580-584 [FREE Full text] [Medline: 9357692]

3. Patrick J, Sabbagh M, Jain S, Zheng H. Spelling correction in clinical notes with emphasis on first suggestion accuracy. In: Proceedings of 2nd Workshop on Building and Evaluating Resources for Biomedical Text Mining (BioTxtM2010). 2010 Presented at: 2nd Workshop on Building and Evaluating Resources for Biomedical Text Mining; 2010; Malta.

4. Lai KH, Topaz M, Goss FR, Zhou L. Automated misspelling detection and correction in clinical free-text records. J Biomed Inform 2015 Jun;55:188-195 [FREE Full text] [doi: 10.1016/j.jbi.2015.04.008] [Medline: 25917057]

5. Fivez P, Šuster S, Daelemans W. Unsupervised Context-Sensitive Spelling Correction of English and Dutch Clinical Free-Text with Word and Character N-Gram Embeddings. Computational Linguistics in the Netherlands Journal 2017;7:39-52 [FREE Full text]

6. Fivez P, Šuster S, Daelemans W. Unsupervised Context-Sensitive Spelling Correction of Clinical Free-Text with Word and Character N-Gram Embeddings. In: Proceedings of the BioNLP 2017 Workshop. 2017 Presented at: BioNLP 2017 Workshop; August 4, 2017; Vancouver, Canada p. 143-148 URL: https://www.aclweb.org/anthology/W17-2317.pdf [doi: 10.18653/v1/w17-2317]

7. Johnson AEW, Pollard TJ, Shen L, Lehman LH, Feng M, Ghassemi M, et al. MIMIC-III, a freely accessible critical care database. Sci Data 2016 May 24;3:160035 [FREE Full text] [doi: 10.1038/sdata.2016.35] [Medline: 27219127]

8. Bojanowski P, Grave E, Joulin A, Mikolov T. Enriching Word Vectors with Subword Information. TACL 2017 Dec;5:135-146 [FREE Full text] [doi: 10.1162/tacl a 00051]

9. Zhang Y, Chen Q, Yang Z, Lin H, Lu Z. BioWordVec, improving biomedical word embeddings with subword information and MeSH. Sci Data 2019 May 10;6(1):52 [FREE Full text] [doi: 10.1038/s41597-019-0055-0] [Medline: 31076572]

10. CDM_ETL-bacteria. GitHub. URL: https://github.com/KU-RIAS/CDM ETL-bacteria [accessed 2021-01-08]

11. Stearns MQ, Price C, Spackman KA, Wang AY. SNOMED clinical terms: overview of the development process and project status. Proc AMIA Symp 2001:662-666 [FREE Full text] [Medline: 11825268]

12. Zhou X, Zheng A, Yin J, Chen R, Zhao X, Xu W, et al. Context-Sensitive Spelling Correction of Consumer-Generated Content on Health Care. JMIR Med Inform 2015 Jul 31;3(3):e27 [FREE Full text] [doi: 10.2196/medinform.4211] [Medline: $\underline{26232246]}$

13. Levenshtein V. Binary codes capable of correcting deletions, insertions and reversals. Soviet Physics Doklady 1966 Feb;10:707.

14. Damerau FJ. A technique for computer detection and correction of spelling errors. Commun. ACM 1964 Mar;7(3):171-176. [doi: $10.1145 / 363958.363994]$

15. Crowell J, Zeng Q, Ngo L, Lacroix E. A frequency-based technique to improve the spelling suggestion rank in medical queries. J Am Med Inform Assoc 2004 Jun;11(3):179-185 [FREE Full text] [doi: 10.1197/jamia.M1474] [Medline: 14764616]

16. Pande H. Effective search space reduction for spell correction using character neural embeddings. In: Proceedings of the 15th Conference of the European Chapter of the Association for Computational Linguistics: Volume 2, Short Papers. 2017 Presented at: 15th Conference of the European Chapter of the Association for Computational Linguistics; April 2017; Valencia, Spain p. 170-174 URL: https://www.aclweb.org/anthology/E17-2027.pdf [doi: 10.18653/v1/e17-2027]

17. How to Write a Spelling Corrector. Norvig. URL: http://norvig.com/spell-correct.html [accessed 2021-02-16]

18. SymSpell. GitHub. URL: https://github.com/wolfgarbe/SymSpell [accessed 2021-02-16]

19. SCOWL (And Friends). Wordlist. URL: http://wordlist.aspell.net/ [accessed 2021-02-16]

20. Nazir A, Qamar U, Zafar I, Shaheen A, Fatima A, Maqbool B. Automated misspelling detection and correction in clinical free-text records. 2018 Presented at: 2018 International Conference on Artificial Intelligence and Big Data (ICAIBD); May 26-28, 2018; Chengdu p. 277-280. [doi: 10.1109/icaibd.2018.8396209]

21. Dorland. Dorland's Dictionary of Medical Acronyms and Abbreviations - 7th Edition. Amsterdam: Elsevier; 2015.

\section{Abbreviations}

CT: clinical terms

ETL: extract-transformation-load

MeSH: Medical Subject Headings

NER: named entity recognition

OOV: out-of-vocabulary 
SNOMED: Systematized Nomenclature of Medicine

SUSC: similarity-based unsupervised spelling correction

Edited by G Eysenbach; submitted 07.11.20; peer-reviewed by $Q$ Chen; comments to author 02.12.20; revised version received
12.01.21; accepted 20.01.21; published 22.02.21
Please cite as:
Kim T, Han SW, Kang M, Lee SH, Kim JH, Joo HJ, Sohn JW
Similarity-Based Unsupervised Spelling Correction Using BioWordVec: Development and Usability Study of Bacterial Culture and
Antimicrobial Susceptibility Reports
JMIR Med Inform 2021; $9(2): e 25530$
URL: $\underline{\text { https://medinform.jmir.org/2021/2/e25530 }}$
doi: $\underline{10.2196 / 25530}$
PMID: $\underline{3616536}$

(CTaehyeong Kim, Sung Won Han, Minji Kang, Se Ha Lee, Jong-Ho Kim, Hyung Joon Joo, Jang Wook Sohn. Originally published in JMIR Medical Informatics (http://medinform.jmir.org), 22.02.2021. This is an open-access article distributed under the terms of the Creative Commons Attribution License (https://creativecommons.org/licenses/by/4.0/), which permits unrestricted use, distribution, and reproduction in any medium, provided the original work, first published in JMIR Medical Informatics, is properly cited. The complete bibliographic information, a link to the original publication on http://medinform.jmir.org/, as well as this copyright and license information must be included. 